\title{
OPINION
}

\section{Risk homeostasis theory: an overview}

\author{
Gerald J S Wilde
}

There is an odd coexistence between two conflicting safety policies that may well be pursued by the same accident prevention agency. The first seeks to improve safety by alleviating the consequences of risky behaviour. It may take the form of seat belt installation and wearing, airbags, crashworthy vehicle design, or forgiving roads (collapsible lamp posts and barriers). This policy offers forgiveness for a moment of inattention or carelessness. The second policy seeks to improve safety by making the consequences of imprudent behaviour more severe and includes things such as speed bumps, narrow street passages, and fines for violations. Here, people are threatened into adopting a safe behaviour; a moment of inattention or carelessness may have a dire outcome.

While these two policies seem logically contradictory, neither is likely to reduce the injury rate, because people adapt their behaviour to changes in environmental conditions. Both theory and data indicate that safety and lifestyle dependent health is unlikely to improve unless the amount of risk people are willing to take is reduced.

\section{Choice of denominator}

In any discussion about injury prevention the criterion of success should be clearly specified, or else confusion abounds. ${ }^{12}$ What is it that we want to achieve: fewer accidents per unit distance driven? Per hour of exposure to traffic? Or per head of population per year?

Sometimes the choice of denominator is obvious. We wish to reduce the number of suicides per head of population, not per pistol or $\mathrm{km}$ of available rope. Success in promoting electrical safety is not measured in terms of fewer cases of electrocution per kwh consumed.

In the domain of traffic, do we want to provide more mobility per case of death or injury, or do we want fewer cases of death and injury? That these two measures of success are not interchangeable is clearly demonstrated by data from the US. Between 1927 and 1987, the death rate per $\mathrm{km}$ driven fell by a factor of about 9 , but the death rate per 100000 inhabitants showed no clear secular trend, neither upward nor downward. Similarly, during a period of steady economic growth, between 1955 and 1972, Ontario saw a major reduction in the traffic death rate per unit distance driven, but an increase per capita. ${ }^{3}$ From a public health point of view, the injury rate per head of population is the most relevant, and that is why that denominator will be used here.

\section{Effect of the business cycle on injuries}

Traffic injury rates show major fluctuations from one time period to another, and it has been established that these fluctuations go hand in hand with the business cycle: in years of high unemployment and low industrial production the per capita death rate is low. ${ }^{4}$

It may be argued that the increased traffic death rate in periods of relative economic prosperity is due to an increased inclination of people to expose themselves to risk on the road. In these periods, more money is to be made by more and faster mobility while the costs of gasoline and car repairs relative to dispensable income are reduced. In depressed economies the death rate on the road is reduced because both amount and riskiness of driving fall for the opposite reasons.

\section{Accepted level of injury risk}

Besides macroeconomic influences, there are other factors that influence the level of accepted risk; these are of a cultural, social, or psychological kind. In general, the amount of risk that people are willing (in fact, prefer) to take can be said to depend on four utility factors and will be greater to the extent that factors 1 and 4 are higher, and factors 2 and 3 are lower:

(1) The expected benefits of risky behaviour alternatives (examples: gaining time by speeding, fighting boredom, increasing mobility).

(2) The expected costs of risky behaviour alternatives (examples: speeding tickets, car repairs, insurance surcharges).

(3) The expected benefits of safe behaviour alternatives (examples: insurance discounts for accident-free periods, enhancement of reputation of responsibility).

(4) The expected costs of safe behaviour alternatives (examples: using an uncomfortable seat belt, being called a coward by one's peers, time loss).

The level of risk at which the net benefit is expected to maximize is called the target level of risk in recognition of the realization that people do not try to minimize risk (which would be zero at zero mobility), but instead attempt to optimize it. ${ }^{3}$ Risk homeostasis theory posits that people at any moment of 


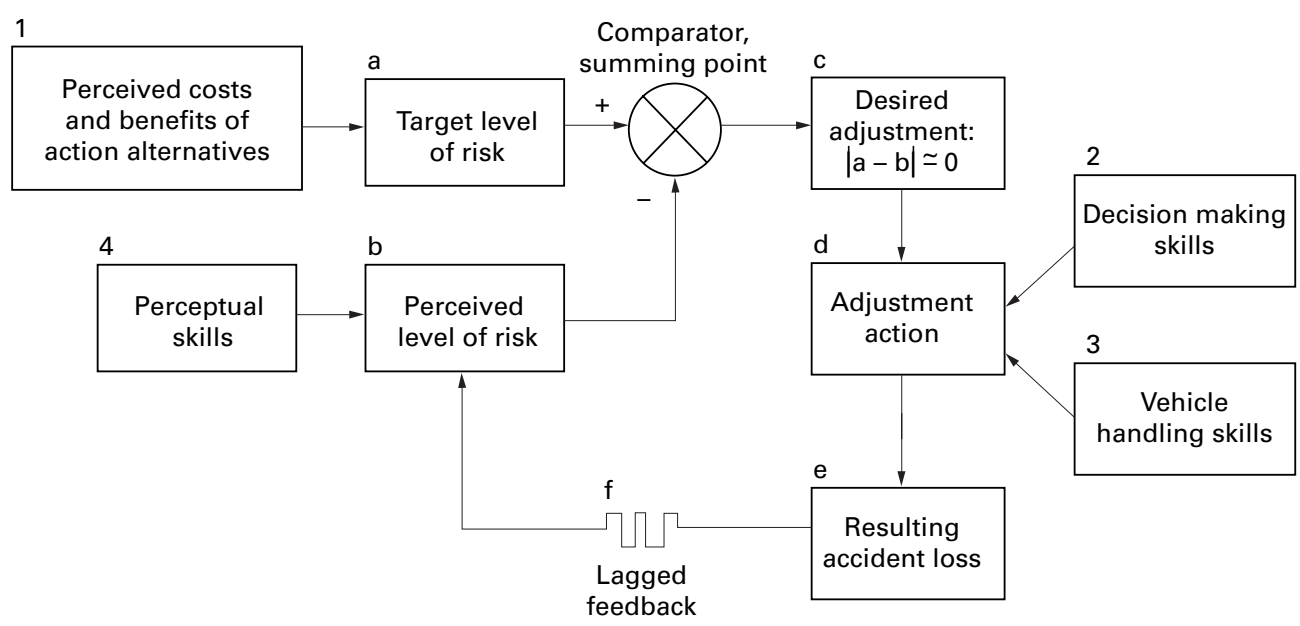

Figure 1 Homeostatic mechanism.

time compare the amount of risk they perceive with their target level of risk and will adjust their behaviour in an attempt to eliminate any discrepancies between the two. Each action carries a certain level of injury likelihood such that the sum total of all actions taken by people over one year explains the accident rate for that year. This rate, in turn, has an effect on the level of risk that people perceive and thus upon their subsequent decisions, and so forth.

This homeostatic mechanism is depicted in fig 1 and constitutes a case of circular causality: a change in the degree of caution displayed in behaviour brings about a change in the injury rate, while a change the injury rate also leads to a change in behaviour. The phenomenon is similar to a thermostat: this instrument controls the actions of the heating/cooling unit which controls the temperature and this in turn controls the actions of the thermostat. There will be fluctuations in the room temperature, but averaged over time, the temperature will remain stable, unless the thermostat is set to a new target (set point) level.

Similarly, the target level of risk is seen as the controlling variable in the causation dynamic of the injury rate. It follows that the basic strategy of injury prevention should be to reduce the level of risk that people are willing to accept.

Because of the closed loop nature of the control process, all other variables, such as variations in skill or environmental conditions can only produce minor and/or short term fluctuations, and these are often reduced or virtually eliminated through anticipatory adaptation ("feed forward control").

\section{Evidence of risk homeostasis}

In the fall of 1967 Sweden changed over from left hand to right hand traffic. This was followed by a marked reduction in the traffic fatality rate. About a year and a half later, the accident rate returned to the trend before the changeover. In terms of fig 1, what happened was a sudden surge in box $b$ as a result of the changeover coming into effect. Perceived risk (box b) was suddenly significantly higher than the target level of risk (box a). Road users adjusted their behaviour by choosing much more prudent behaviour alternatives (box d). As a result, the fatal injury rate dropped (box e). After some time (f), however, people discovered-through the mass media as well as their own experiences - that the roads were not as dangerous as they had thought they were. The level of perceived risk dropped and less often exceeded the target level of risk. Consequently, road users opted for less cautious behaviour alternatives and the fatal injury rate rose again. ${ }^{3}$

Numerous other findings can be explained by risk homeostasis theory. In road sections where the accident rate per $\mathrm{km}$ driven is low, drivers move faster. Mandatory wearing of seat belts reduces the likelihood of death or injury in case an accident happens, but does not reduce the death rate per capita. ${ }^{3}$ Similarly, "... airbag equipped cars tend to be driven more aggressively and that aggressiveness appears to offset the effect of the airbag for the driver and increases the risk of death to others". ${ }^{6}$ Cars outfitted with antilock brakes are driven faster, more carelessly, and closer to the car in front, braked more abruptly, and have no lower accident rate per hour of exposure than cars without these devices. ${ }^{378}$ Similarly, with better road lighting motorists drive faster and pay less attention. ${ }^{9}$

Driver training or a mandatory course of driving on slippery roads does not reduce accident risk. ${ }^{3}{ }^{10}$ Such training does indeed improve skill, but it apparently increases confidence even more, with the end effect that driver education graduates show a higher accident rate per capita.

The introduction of childproof medicine vials has failed to limit the number of cases of accidental poisoning. These, in fact, became more frequent, apparently as the result of parents becoming less careful in the handling and storing of the "safer" bottles. ${ }^{10}$ A recent Swedish study showed that the more traffic safety education children in kindergarten and primary school had received, the higher their traffic injury rate. This was attributed to the greater independence and mobility, including 
the use of a bicycle, that better trained children were allowed. ${ }^{12}$

\section{Incentives for safety}

On the other hand, incentive systems for accident-free operation have been shown to be a very powerful method for the reduction of injury rates. Incentives, that is, future rewards contingent upon fulfilling a future condition, increase the perceived benefits of safe behaviour alternatives (utility factor 3 above). There have been many studies of the effectiveness of incentive schemes, both in industrial settings and traffic and their most productive features have been identified. Injury rate reductions ranging from 10 to $90 \%$ have been observed. The only undesirable side effect noted so far is the underreporting of accidents, but this phenomenon is limited to relatively minor injuries and property damage. Among their positive side effects is a more congenial social climate. ${ }^{3}$

The remarkable effectiveness of incentive programmes is arguably due to the fact that they enhance people's perceived value of the future. The prospect of future gratification causes people to look forward to the future with positive expectation. They will thus have a greater desire to be alive and well when that future comes (utility factor 3 above), and be more inclined to take action to protect their health and safety. And indeed, there is evidence accumulating that individuals who are marked by a high valuation of the future relative to the present display fewer unsafe behaviours and unhealthy lifestyles. ${ }^{13}$ In a recent Canadian study of late adolescents and early adults, a significant relation was found between safe driving practices, regular seat belt use, and moderate alcohol consumption on the one hand and a high valuation of the future on the other. ${ }^{14}$

\section{Conclusion}

The theory of risk homeostasis (also known as "risk compensation") was primarily developed and validated in the area of road safety. Some of the supporting data, however, come from quite different behaviour domains including smoking and settling in flood prone territories. This is not surprising because the mechanisms that are involved in risk homeostasis are probably universal. Moreover, the accident prevention strategy that follows from risk homeostasis theory has been found effective in many areas. Incentives for safety and health may be viewed as one example of a wider class of (not technological but) "expectationist" interventions which offer people more positive anticipations regarding their future than is currently the case and thus motivate them to be more cautious with life and limb.

1 Wilde GJS. On the choice of denominator for the calculation of accident rates. In: Yagar S, ed. Transport risk assessment. Waterloo, Ontario: University of Waterloo Press, 1994:139-54

2 Wilde GJS. Risk homeostasis theory and traffic accidents: propositions, deductions and discussion of dissension in recent reactions. Ergonomics 1988;31:441-68.

3 Wilde GJS. Target risk: dealing with the danger of death, disease and damage in everyday decisions. Toronto: PDE Publications, 1994

4 Wilde GJS, Simonet SL. Economic fluctuations and the traffic accident rate in Switzerland: a longitudinal perspective. Berne: Swiss Council for Accident Prevention, 1996.

5 Wilde GJS. The theory of risk homeostasis: implications for safety and health. Risk Analysis 1982;2:209-25.

6 Peterson S, Hoffer G, Millner E. Are drivers of air-bagequipped cars more aggressive? A test of the offsetting behavior hypothesis. Fournal of Law and Economics 1995;38: 251-64.

7 Fosser S, Sageber F, Sætermo AF. Behavioural adaptation to antilock brakes-drivers keep a shorter distance. Nordic Road and Transport Research 1996;8:21-2.

8 Grant BA, Smiley A. Driver response to antilock brakes: a demonstration of behavioural adaptation. Proceedings of the Canadian Multidisicplinary Road Safety Conference VIII. University of Saskatoon, Saskatchewan, Canada, 14-16 June 1993.

9 Björnskau T, Fosser S. Road lighting increases safety-but motorists drive slightly faster and pay less attention. Nordic Road and Transport Research 1996;8:20.

10 Christensen P, Glad A. Mandatory course of driving on slippery roads does not reduce the accident risk. Nordic Road and Transport Research 1996;8:22-3.

11 Viscusi WK. The lullling effect: the impact of child-resistant packaging on aspirin and analgesic ingestions. American Economic Review 1984;74:324-7.

12 Johansson BS. Trafiktränade barn löper större olycksrisk. Väg-och Transportforskningsinstitutet Aktuellt 1997;4(June): 9.

13 Björgvinsson T, Wilde GJS. Risky health and safety habits related to perceived value of the future. Safety Science 1996; 22:27-33.

14 Björgvinsson T. Health and safety habits as a function of the perceived value of the future. [Doctoral dissertation.] Kingston, Ontario: Department of Psychology, Queen's University, 1998.

\section{Notice to subscribers}

We regret that a binding error occurred at our printer and some copies of the journal have pages 17-32 missing and 33-48 duplicated. Would any subscriber who finds that they have a faulty copy please contact Sue Heels at Injury Prevention, BMA House, Tavistock Square, London WC1H 9JR, UK and it will be replaced (fax: +44 (0)171 383 6668). 